\title{
Procesos espaciales asociados a la construcción de la doble calzada BTS en los municipios de Tunja, Cómbita y Oicatá (2005 2012) ${ }^{1}$
}

Spatial Processes Associated with the Construction of the Dual Highway BTS in the Municipalities of Tunja, Oicatá and Cómbita (2005 2012)

Andrés Felipe Bautista Vargas ${ }^{2}$ Flor Ángela Cerquera Escobar ${ }^{3}$

Para citar este artículo, utilice el nombre completo así:

Bautista, A. F. \& Cerquera, F. A. (2014). Procesos espaciales asociados a la construcción de la doble calzada BTS en los municipios de Tunja, Cómbita y Oicatá (2005-2012). Perspectiva Geográfica, 19(2), 219-240

\section{Resumen}

Se aborda el estudio de los procesos espaciales asociados a la construcción de la infraestructura vial de la doble calzada Briceño-Tunja-Sogamoso BTS, en los municipios de Tunja, Cómbita y Oicatá, en Colombia, a través de la fotointerpretación de imágenes Spot 2005 y Google Map Tiff 2012, aplicando la lectura de la leyenda nacional de coberturas de la tierra metodología Corine Land Cover (CLC) adaptación para Colombia escala 1:100.000 (Ministerio de Ambiente y Desarrollo Sostenible, 2010). Se advirtieron diferentes procesos espaciotemporales no lineales, que obedecen a la incidencia de la infraestructura vial en la localización de nuevo tejido urbano discontinuo, fragmentación

\footnotetext{
1 Este artículo corresponde al proyecto "Transformaciones en el territorio de los municipios de Tunja, Cómbita y Oicatá asociados a la infraestructura vial de la doble calzada BTS, bajo un análisis regional y local 2005-2012".

2 Licenciado en Ciencias Sociales de la Universidad Pedagogica y Tecnologica de Colombia. Magíster en Geografia convenio Universidad Pedagogica y Tecnologica de Colombia e Instituto Geográfico Agustín Codazzi. bautistaandresfelipe@gmail.com.

3 Ingeniero de Transporte y Vías. Magíster en Ingeniería de Tránsito y Transporte. Doctora en Geografía UPTC-IGAC. Docente investigador UPTC. faceitv@uptc.edu.co
} 
predial, y cambio de coberturas en su área de influencia. Como resultado se obtuvo un análisis espaciotemporal 2005-2012, conjugado con la estimación de la valorización predial, el cual resulta adecuado para apreciar la incidencia en el área de influencia, como evidenciar cambios transicionales en el uso del suelo.

Palabras clave: Corine Land Cover-CLC, infraestructura vial, procesos espaciales asociados, territorio, transporte.

\begin{abstract}
The study of spatial processes associated with the construction of road infrastructure of the dual highway Briceño - Tunja - Sogamoso BTS, in the municipalities of Tunja, Cómbita and Oicata in Colombia is addressed. Through photo-interpretation of images from Spot 2005 and Google Map Tiff 2012, applying the reading of the national legend of land cover, using the Corine Land Cover (CLC) methodology, adapted for Colombia 1: 100,000 (Ministry of Environment and Sustainable Development, 2010). Different nonlinear spatiotemporal processes were observed that obey the incidence of road infrastructure in locating new discontinued urban fabric, farm fragmentation and change of coverage in their area of influence. The result is a spatiotemporal analysis 2005-2012, combined with the estimate of property developments, which is suitable for assessing the impact on the area of influence, as evidenced by transitional changes in land use.
\end{abstract}

Keywords: Corine Land Cover-CLC, road infrastructure, associated processes, land, transportation. 


\section{Introducción}

El desarrollo de proyectos de infraestructura vial es un renglón de interés a la luz de la política de integración nacional del país. Ampliar los niveles de competitividad a través del servicio y construcción de la red nacional de carreteras, es uno de los propósitos de la actual política de transportes, según el Consejo Nacional de Política Económica y Social (Consejo Nacional de Política Económica y Social -CONPES-, 2004). La dotación de infraestructuras viales no solo ofrece ventajas comparativas en aquellos territorios al recibir mejoras en cuanto a la conectividad, sino que es un elemento de transporte potencialmente dinámico al generar grandes expectativas donde es incorporada (Cerquera, 2013).

Estas expectativas, según Bellet (2013), tardan mucho tiempo en materializarse, y si lo hacen, se debe tener en cuenta que son procesos espaciotemporales no lineales a su construcción. Los proyectos de infraestructura vial son obras que tienen un elevado carácter espacial, por lo tanto estimulan cambios en el espacio con intensidades y frecuencias distintas, evidenciados en transformaciones físicas del territorio y relativos al uso del suelo, los mismos que han sido tratados desde perspectivas de tipo causal y de con- formidad con el impacto generado en el territorio (Blanco, 2006).

Estas perspectivas, aun cuando prevalecen como líneas de estudio de los efectos del transporte, también pueden ser consideradas como procesos espaciales asociados, dada la complejidad de determinar si la infraestructura es el elemento responsable de dichos cambios (Offner, 1993; Millares-Guach, 2002; Governa, 2007; Garmendia, 2008). Pues las infraestructuras viales "son elementos de transporte polivalentes, es decir, instaurados con múltiples propósitos" (Urbano, 1993, p. 89). Esto significa que su acción no es estática, sino que propugna una dinámica en el espacio, relativa y acorde con su función social y económica, por lo tanto, representa no un bien de consumo final en el proceso de transporte, sino un bien intermedio permisivo para la articulación y circulación de bienes que son transportados (Izquierdo, 1994).

La doble calzada BTS no solo es una infraestructura vial circunscrita a las anteriores consideraciones, sino que representa uno de los corredores viales de competitividad nacional vinculada al programa de tercera generación de concesiones viales del país, cuya funcionalidad es representativa en términos de articulación para los municipios y departamentos en donde cursa su construcción. De este se esperan impactos de gran envergadura 
en las áreas urbanas, suburbanas y rurales de los municipios que influencia directamente. El proyecto consta de 18 tramos localizados entre los departamentos de Cundinamarca y Boyacá, Colombia, donde uno de estos tramos lo conforma la variante de doble calzada en el municipio de Tunja, corredor seleccionado como área de estudio, analizado dentro de un marco localizado en el que fácilmente se visualizan cambios sobre el espacio físico rural principalmente.

Algunos de los indicadores lo relacionan con cambios en las coberturas, dinámicas de valorización, localización de tejido urbano discontinuo y fragmentación predial en aquellas áreas inmediatas al paso de la infraestructura vial. A partir de estos indicadores se obtiene una descripción multitemporal 2005-2012 del área de influencia de la infraestructura vial en los municipios de Tunja, Cómbita y Oicatá, a fin de ofrecer conocimiento específico de las dinámicas espaciales que pudieron asociarse a su construcción, el cual resulta adecuado para estimar metodológica y conceptualmente contribuciones importantes de la incidencia del transporte en el territorio.

\section{Localización geográfica y carac- terísticas del área de estudio}

El área de estudio de la infraestructura vial se encuentra ubicada en el municipio de Tunja, con áreas compartidas entre los municipios de Oicatá y Cómbita del departamento de Boyacá, Colombia. Cuenta con un área superficial de $8,75 \mathrm{~km}^{2}$ caracterizada por su riqueza paisajística, con una longitud aproximada de $17.800 \mathrm{~m}$, los cuales se encuentran en pavimento flexible y sus obras cumplen con las especificaciones de variante de doble calzada, con el propósito de descongestionar el área urbana de Tunja y flexibilizar el transporte terrestre de Bogotá a Sogamoso y viceversa.

\section{Metodología}

Para identificar los procesos espaciales asociados de la infraestructura vial en los municipios de Tunja, Cómbita y Oicatá, se efectuaron dos procesos. El primero corresponde a la interpretación de imágenes Spot 2005 y retrointerpretación Google Maps Tiff 2012 del área de influencia de la vía definida a 0,25 ha. Esta área tenía un planeamiento anterior realizado por la obra de construcción, lo que facilitó el recorrido y el acceso para la toma en los puntos de control de mayor importancia e incidencia dentro de la longitud de la BTS enmarcada en el área de estudio. 


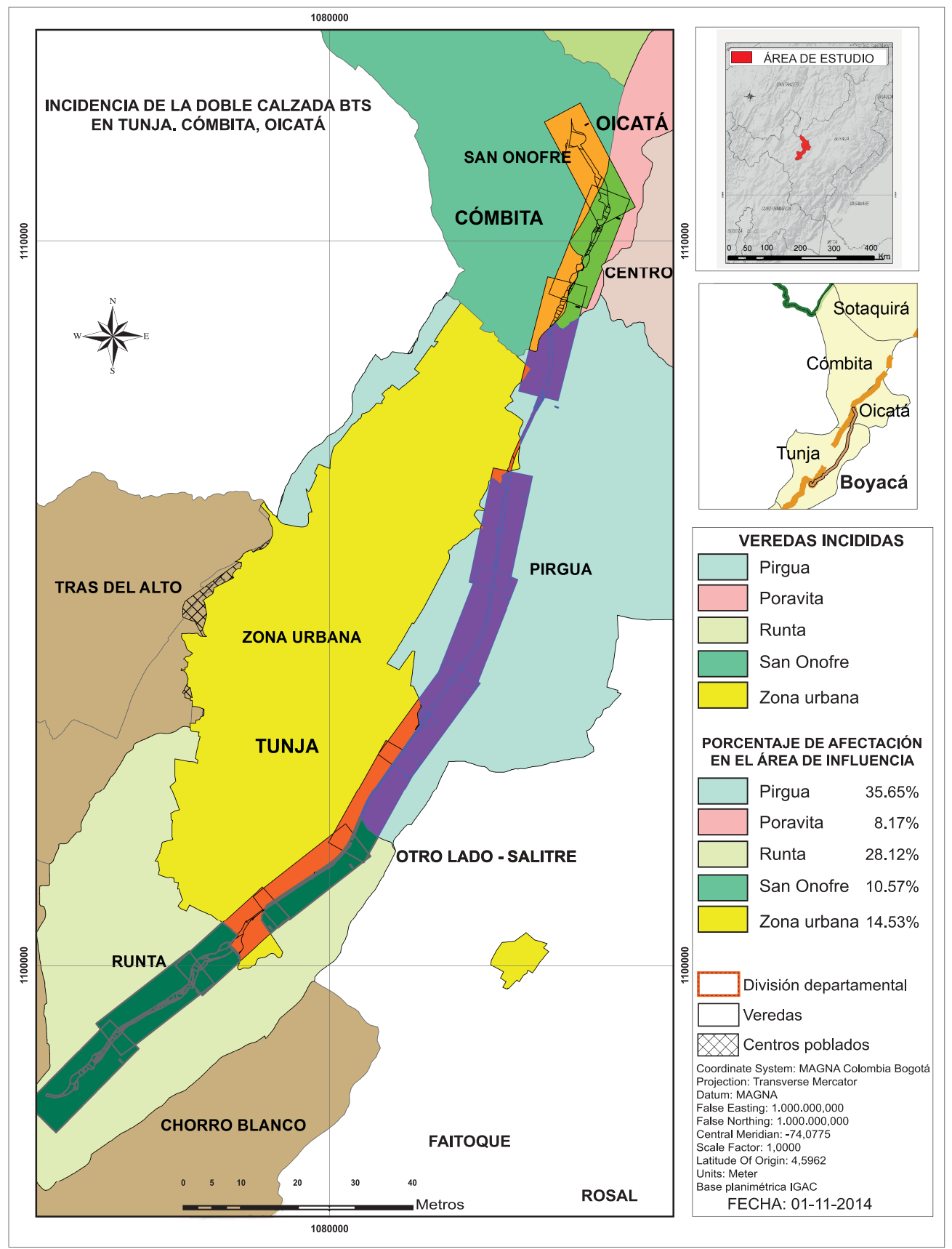

Figura 1. Localización del área de estudio y porcentajes de incidencia sobre los municipios de Tunja, Cómbita y Oicatá

Fuente: elaboración propia a partir de la cartografía digital del Plan de Ordenamiento Territorial de Tunja 2001. 


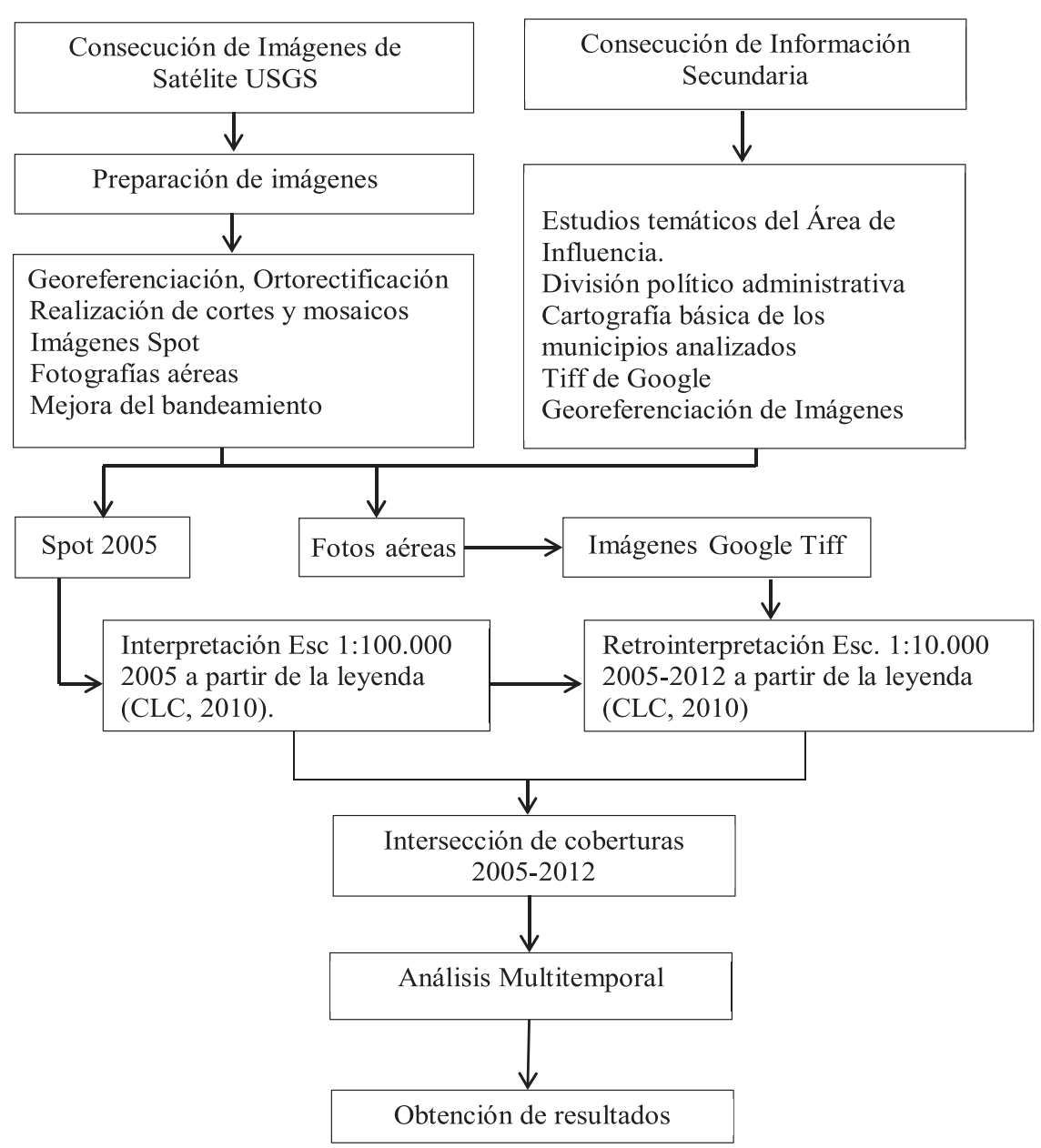

Figura 2. Proceso metodológico

Fuente: elaboración propia, estructurado a partir de Corredor, Cárdenas y Ordoñez, (2011, p.157).

Para efectos del segundo proceso, el cual corresponde a la proyección de valorización predial del área de influencia de la vía en los municipios de Tunja, Cómbita y Oicatá, se tuvieron en cuenta los aspectos biofísicos según el mapa de zonas físicas y geoeconómicas de los municipios de estudio, registro de predios vigencia 2005 de la Subdirección de Catastro del (IGAC), y la metodología del índice de valorización predial IVP, del Departamento Administrativo Nacional de Estadística (Departamento Administrativo Nacional de Estadística -DANE-, 2013). 
2.1 Incidencia espacial en el área inmediata a la infraestructura vial de la doble calzada BTS en los municipios de Tunja, Cómbita y Oicatá

La incidencia espacial por la construcción de la infraestructura vial ha sido notable, particularmente en las zonas rurales y suburbanas de los municipios en estudio, donde el
$85 \%$ de afectación espacial se originó en las zonas rurales y el restante en zona urbana. Dicha afectación se ha generado específicamente en el municipio de Tunja, principalmente en las veredas de Pirgua y Runta, donde se llevan a cabo actividades de uso predominantemente agrícolas, de acuerdo con las directrices del plan de ordenamiento territorial del municipio de Tunja POT (2001).

Tabla 1. Estadística de área de incidencia en los municipios por vereda

\begin{tabular}{|c|c|c|c|c|c|c|c|c|}
\hline Ítem & Sector & $\begin{array}{c}\text { Munici- } \\
\text { pio }\end{array}$ & Vereda & $\begin{array}{c}\mathbf{N}^{\circ} \text { de predios } \\
\text { afectados }\end{array}$ & $\begin{array}{c}\text { Área total de } \\
\text { predios en } \\
\text { el municipio } \\
/ \text { Ha }\end{array}$ & $\begin{array}{c}\text { Área de } \\
\text { predios en la } \\
\text { vereda /Ha }\end{array}$ & $\begin{array}{c}\text { Área de } \\
\text { afectación } \\
\text { predial en la } \\
\text { vereda / Ha }\end{array}$ & $\%$ \\
\hline 1 & Rural & Tunja & $\begin{array}{c}\text { Chorro } \\
\text { Blanco }\end{array}$ & 107 & - & 1.576 & 2.499 & 2.86 \\
\hline 2 & Rural & Tunja & Runta & 260 & - & 1.376 & 246 & 28.12 \\
\hline 3 & Rural & Tunja & Pirgua & 350 & t- & 1.890 & 311 & 35.65 \\
\hline 4 & Urbano & Tunja & $\begin{array}{c}\text { Zona } \\
\text { Urbana }\end{array}$ & 280 & - & 1.626 & 127 & 14.53 \\
\hline 5 & Rural & Oicatá & $\begin{array}{c}\text { Pora- } \\
\text { vita }\end{array}$ & 180 & 5.941 & 904 & 72 & 8.27 \\
\hline 6 & Rural & Cómbita & $\begin{array}{c}\text { San } \\
\text { Onofre }\end{array}$ & 136 & 14.622 & 1.083 & 92 & 10.57 \\
\hline & & & Total & 1.313 & 32.583 & 14.925 & 4.057 & 100 \\
\hline
\end{tabular}

Fuente: elaboración propia a partir de estudio multitemporal 2005-2012.

Según la Tabla 1, esta infraestructura incidió en seis veredas y en 1.313 predios, lo cual es considerable, especialmente en la zona urbana de Tunja y en la vereda de San Onofre del municipio de Cómbita, que por causa del trazado de la vía refieren mayor incidencia respecto a las demás veredas. Hay que tener en cuenta que la localización de la infraestructura afectó el $81 \%$ en el área del municipio de Tunja, del $85 \%$ del área establecida, por la incidencia espacial de la vía en los tres municipios analizados, donde se observa un marcado fraccionamiento predial en las veredas de San Onofre y Poravita de los municipios de Oicatá y Cómbita, y en las veredas Runta y Pirgua del municipio de Tunja, de acuerdo con la Figura 3. 


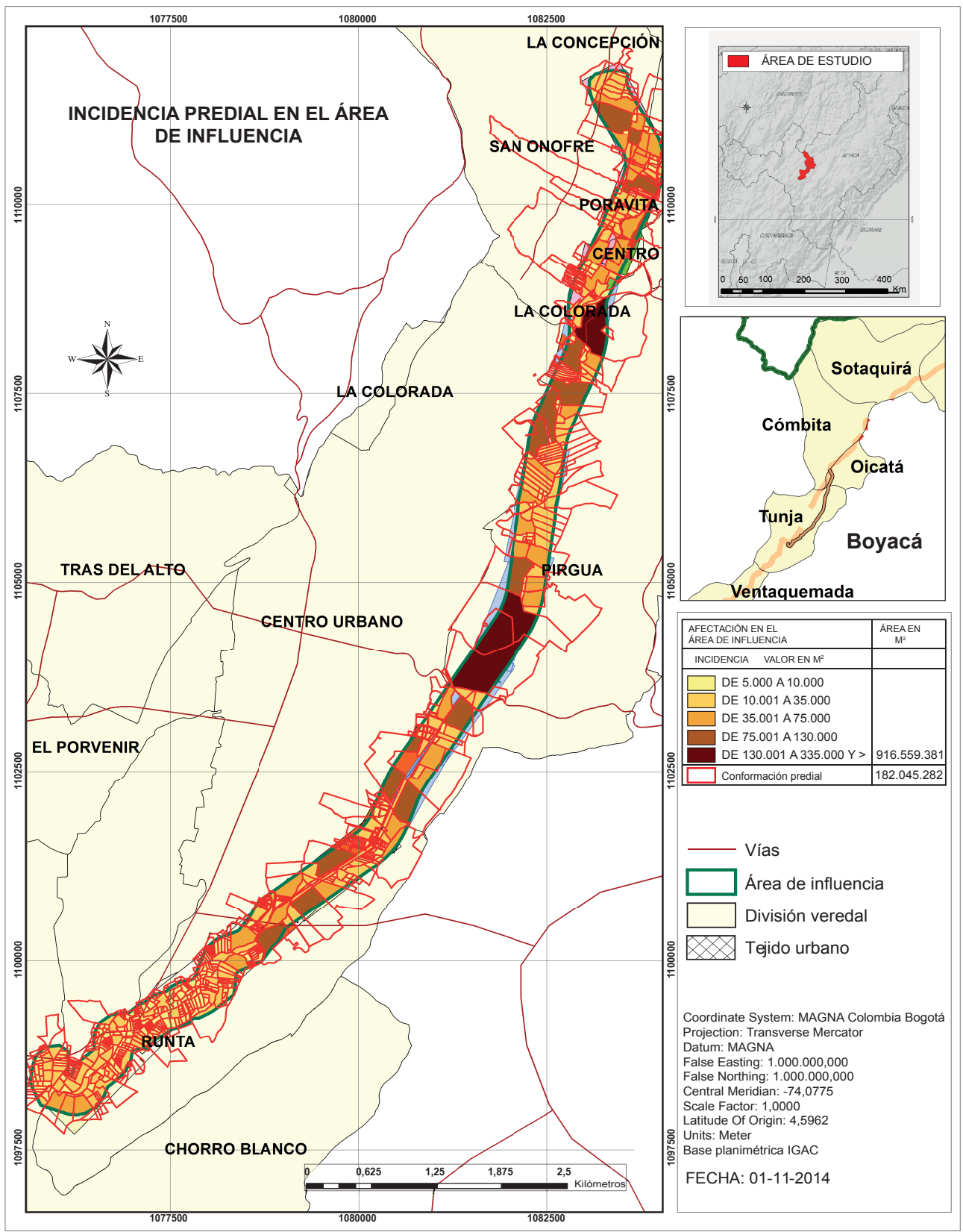

Figura 3. Incidencia predial en el área inmediata de la infraestructura vial en los municipios de Tunja, Cómbita y Oicatá

Fuente: Elaboración propia a partir de vigencia 2005 Catastro (IGAC). 
En efecto, se trata de una vía de carácter nacional de doble calzada que tiene como propósito no solo mejorar la conectividad a su paso por los municipios, sino descongestionar el tráfico vehicular que supeditaba la vía central urbana de la ciudad de Tunja. Se espera que su dinámica tenga una influencia importante en el área inmediata. Esta dinámica se ve reflejada en áreas de mayor afectación predial, dadas las condiciones de parcelamiento, como se presenta en la zona suroriental del municipio de Tunja, donde se observa un elevado número de predios involucrados, en cuanto a áreas por metro cuadrado, cuyo valor se encuentra entre los 5.000 y $10.000 \mathrm{~m}^{2}$.

Esto quiere decir, en términos de afectación predial, que el grado de incidencia de la vía fue de mayor proporción en áreas de menor tamaño que en aquellas de mayor área. Esto se debe al ordenamiento parcelario de las veredas, como a las mismas zonas prediales de menor, mediano y mayor tamaño, cuyas características de localización no solo determinaron la dimensión de la afectación predial, sino estimularon algunos procesos espaciales asociados a nuevos usos del suelo y valorización predial, como se evidencia en la Figura 4.

\subsection{Dinámica en la valorización} predial en el área de influencia inmediata de la infraestructura vial a su paso por los municipios de Tunja, Cómbita y Oicatá

Dentro de los procesos espaciales asociados, la incorporación de infraestructuras viales representa en términos de conectividad no solo un elemento básico del transporte, sino que, en virtud de su espacialidad, genera una incidencia en el territorio que depende del grado de desarrollo, tanto de los centros económicos y urbanos que conecta, como de aquellas áreas que por su localización resultan beneficiadas de su servicio (Cruz, 2011).

Este planteamiento permite afirmar que cualquier incorporación al territorio, en términos de infraestructuras, implica variaciones en los precios del suelo, cuyos atributos potencian de manera específica su valor, pues las unidades prediales no solo representan un conjunto de atributos estructurales como es el tamaño y número, sino características determinadas por su localización, entre las que se destacan, vecindarios, tráfico, equipamientos y acceso vial (Rubio \& Vargas, 2010; Perdomo, 2010).

En el caso de las zonas prediales del área de influencia, se puede observar la definición de tres zonas de valorización, estimadas de acuerdo con el índice de valorización predial (IVP), en consonancia con los precios establecidos para el año 2005, cuya 


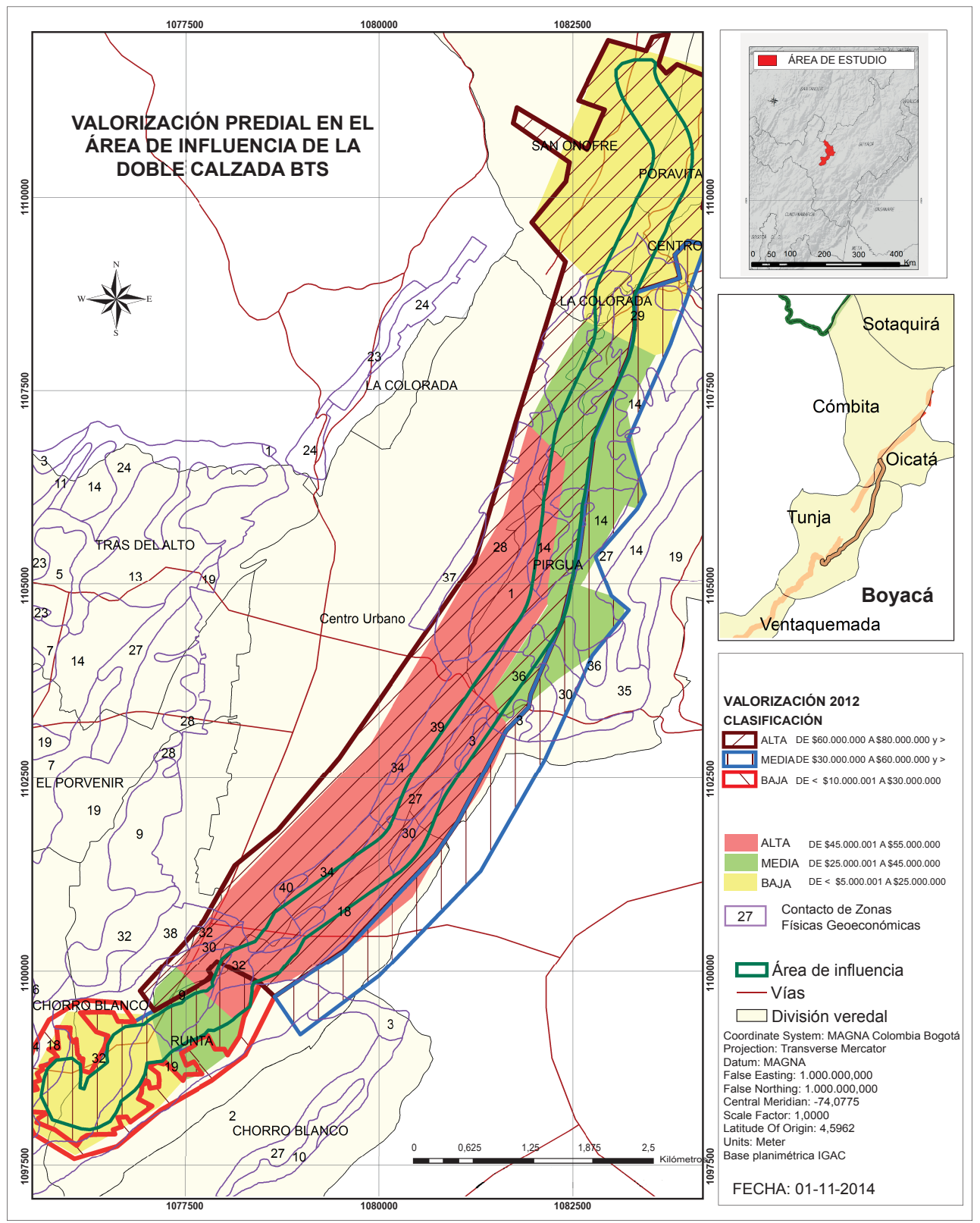

Figura 4. Proyección de la valorización predial en el área de influencia inmediata de la infraestructura vial en los municipios de Tunja, Cómbita y Oicatá

Fuente: elaboración propia a partir de metodología IVP (DANE, 2013). 
clasificación corresponde en primera instancia a la zona central del municipio de Tunja, la cual guarda un valor alto con respecto a los extremos sur y norte, debido a que la zona está articulada a un sistema vial en óptimas condiciones que conduce hacia áreas de orden comercial, de servicios, y de gran densidad residencial. Además, según las zonas geoeconómicas, este sector presenta mejores aptitudes de suelo que las demás zonas prediales.

Sin embargo, en términos de valorización media, se observan dos zonas que por su localización se relacionan con áreas inmediatas a la expansión urbana del municipio de Tunja y de la zona rural del municipio de Oicatá, y desde luego, marcan una diferencia considerable, dado que los valores prediales estimados para estos sectores oscilaron entre 30 y $40 \%$ menos que en las zonas de valorización porcentual alta. Cifras que expresan como dichas zonas prediales configuran importantes valores, cuyos atributos físicos de localización y tamaños prediales influyen tanto en que la afectación predial haya sido de menor proporción que la zona sur, como por implicar predios de mayor tamaño.

Las zonas prediales de menor valor son predios que se localizan en los externos norte y sur de los municipios, y se caracterizan por el parcelamiento, particularmente en la zona sur del municipio de Tunja. Además debe tenerse presente que estas zonas denotan predios de menor tamaño, baja densidad poblacional, y una red vial carreteable sin pavimentar hacia áreas rurales, lo cual indica que por la naturaleza y localización de los predios convergen incipientes desarrollos en torno a la infraestructura vial, y por consiguiente, no expresan en términos de valor un patrón de valorización predial diferente o semejante al de las zonas de mayor valorización, como es el caso de las zonas centrales del área inmediata a la vía.

\subsection{Principales actividades influen- ciadas por la infraestructura vial BTS en los municipios de Tunja, Cómbita y Oicatá}

De acuerdo con el informe inventario socioeconómico (Estudio Socioeconómico, 2011) asentado alrededor del derecho de la vía del año 2011, la construcción de la infraestructura vial de la doble calzada en torno a los municipios de Tunja, Cómbita y Oicatá, incidió aproximadamente en 600 unidades comerciales y de servicios, localizadas en su mayoría en el casco urbano del municipio de Tunja, y en su costado oriental. Este inventario evidenció nacientes actividades sobre el eje vial que ascendieron a 44 , entre las que se pueden destacar estaciones de servicio, campos de deporte extremo, establecimientos de comercio, restaurantes, cuya 
localización es consecuencia de la apertura del nuevo acceso vial, como de la espacialidad ocasionada por la influencia de la vía.

Esta dinámica es explicada como uno de los numerosos efectos asociados que genera la incorporación de una infraestructura de este carácter en el territorio. No solo se concibe una afectación espacial en las diferentes unidades económicas y de servicios localizadas en el área de influencia inmediata de los municipios, sino que también emergen algunas otras actividades que, una vez dadas las condiciones de acceso, se localizan sobre el eje vial beneficiándose de los niveles de servicio y operación vial (Garmendia, Ureña \& Coronado, 2011).

Según Fariña, Lamiquiz y Pozueta (2000), la influencia en las actividades tarda más tiempo en ser manifestada, debido a que estas suponen un cambio profundo en el uso del suelo y en algunas directrices de orden económico para que se originen procesos de ocupación sobre el eje vial. No obstante, para el caso de los municipios influenciados, se observa en la Figura 5 un número importante de polígonos localizados sobre la vía, que corresponden a actividades de tipo extractivo y tejido urbano continuo y discontinuo, según la fotointerpretación realizada a partir de la leyenda CLC, (Ministerio de Ambiente y Desarrollo Sostenible,
2010), y que pueden considerarse como procesos espaciales asociados.

Estos polígonos suman 132, y aproximadamente incidieron 48 ha, discriminadas en áreas rurales $\mathrm{y}$ urbanas de los municipios influenciados, correspondientes a zonas de expansión urbana para el caso de Tunja, y zonas rurales para el caso de los municipios de Cómbita y Oicatá, donde se advierte un número importante de polígonos existentes fuera de la zona urbana, lo cual significa que la construcción de la infraestructura ha influido de forma relevante en la localización de tejido urbano discontinuo, y en el origen de actividades complementarias, como se expresa en la Figura 5 del corredor vial.

Una de las actividades comerciales señaladas, corresponde al sector extractivo, cuya localización es consecuencia de las propiedades del suelo de la zona, y de la articulación de vías de carácter intermunicipal, que ligada a su accesibilidad surge como uno de los procesos espaciales asociados de mayor relevancia en esta parte del territorio municipal. Igualmente se pudo identificar tejido rural edificado, que atañe a aquellas zonas particularmente dispuestas en las áreas rurales de los municipios de Cómbita y Oicatá, y que puede estar directamente asociado con la apertura de accesos viales rurales. 


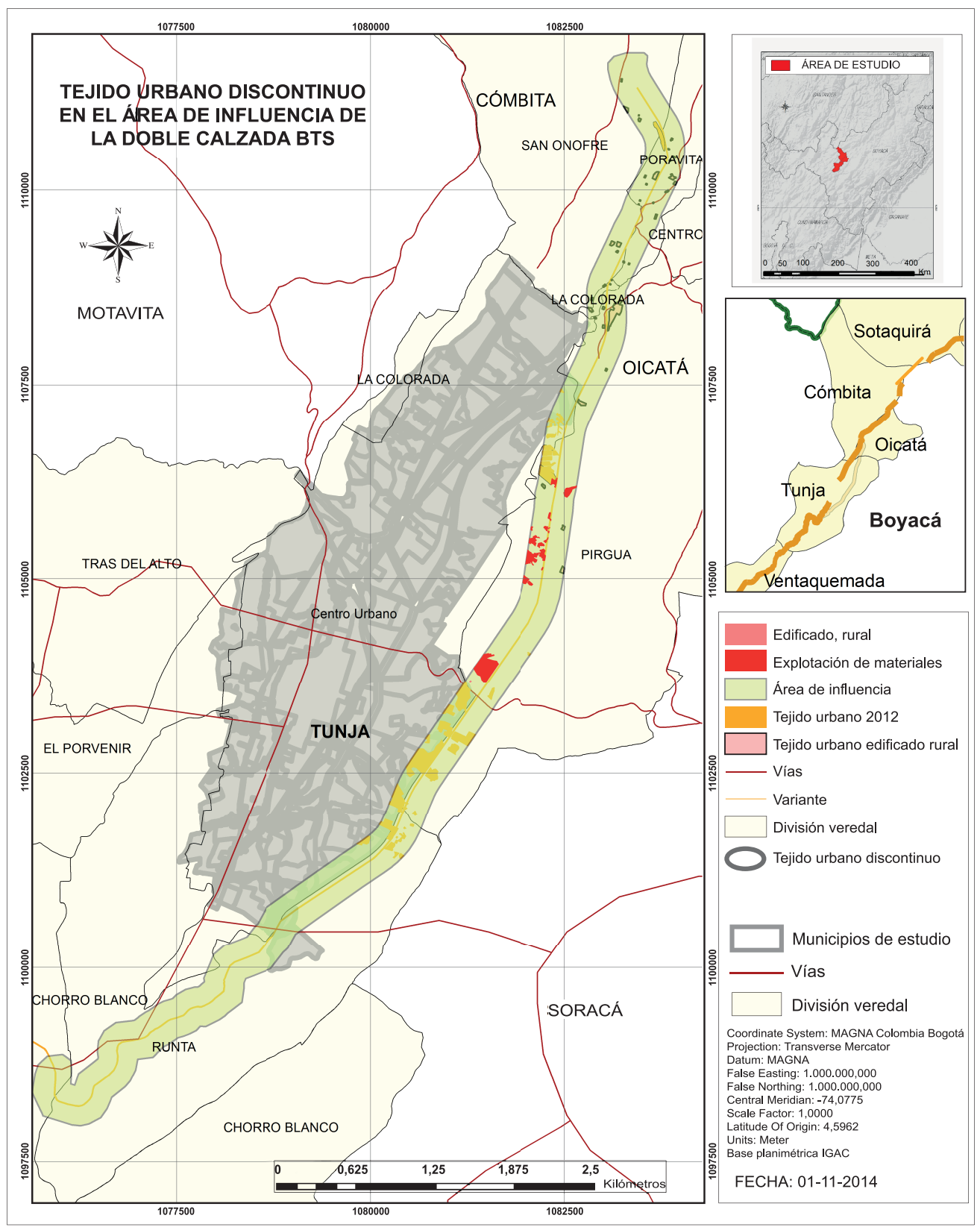

Figura 5. Tejido urbano y actividades localizadas sobre la infraestructura vial Fuente: elaboración propia a partir de estudio multitemporal 2005-2012. 
A la luz de lo expuesto, es natural que la proliferación de tejido urbano continuo se considere como una de las consecuencias que generará, a largo plazo, el paso de la infraestructura vial, y que, por tanto, estimulará el desarrollo de actividades económicas de carácter microlocal, cuya implementación se irá expandiendo de conformidad con el tiempo y la disponibilidad de extensiones de suelo localizadas en el área de influencia, generando cambios transitorios en las coberturas por el paso de la vía.

\subsection{Cambios en las coberturas} en el área de influencia de la infraestructura vial a su paso por

\section{los municipios de Tunja, Cómbita y} Oicatá

Lo anterior permite considerar que el transporte y la infraestructura vial son dos elementos que hacen parte de la estructura espacial del territorio, su incorporación se traduce no solo en cambios paulatinos y relativos al uso del suelo, sino que configura nuevas dinámicas en en el desarrollo espacial localizado en las áreas de influencia. Esto implica una modificación de la articulación vial, así como también un cambio significativo en las coberturas vegetales, como se presenta en la Tabla 2.

Tabla 2. Cobertura del área de influencia 2005, de nivel 3 para una escala 1:100.000

\begin{tabular}{|c|l|c|c|}
\hline Código & \multicolumn{1}{|c|}{ Cobertura CLC 2012 } & Ha & \% \\
\hline 112 & Tejido urbano discontinuo & 0.8601 & 0.1 \\
\hline 231 & Pastos limpios & 297.303 & 33.98 \\
\hline 242 & Mosaico de pastos y cultivos & 305.777 & 34.95 \\
\hline 243 & Mosaico de cultivos, pastos y espacios naturales & 24.020 & 2.83 \\
\hline 333 & Tierras desnudas y degradadas & 246.350 & 28.14 \\
\hline Total & & 873.450 & $100 \%$ \\
\hline
\end{tabular}

Fuente: Elaboración propia a partir de estudio multitemporal 2005-2012.

Según la Tabla 2, el $69 \%$ del área influenciada por la vía para el año 2005 , corresponde a pastos limpios y mosaico de pastos y cultivos, el 28 $\%$ a tierras desnudas y degradadas, y el $3 \%$ comprende tejido urbano discontinuo y mosaico de cultivos, pastos y espacios naturales; atributos que permiten considerar no solo una incidencia físico-rural en la mayor parte del suelo dispuesto para cultivos transitorios y permanentes, sino advertir la fragmentación de espacios naturales y la pérdida de hectáreas que se vieron irreversiblemente afectadas, además de la fragmentación predial y la exposición de una nueva configuración del espacio rural confinando en esta parte del territorio municipal. 


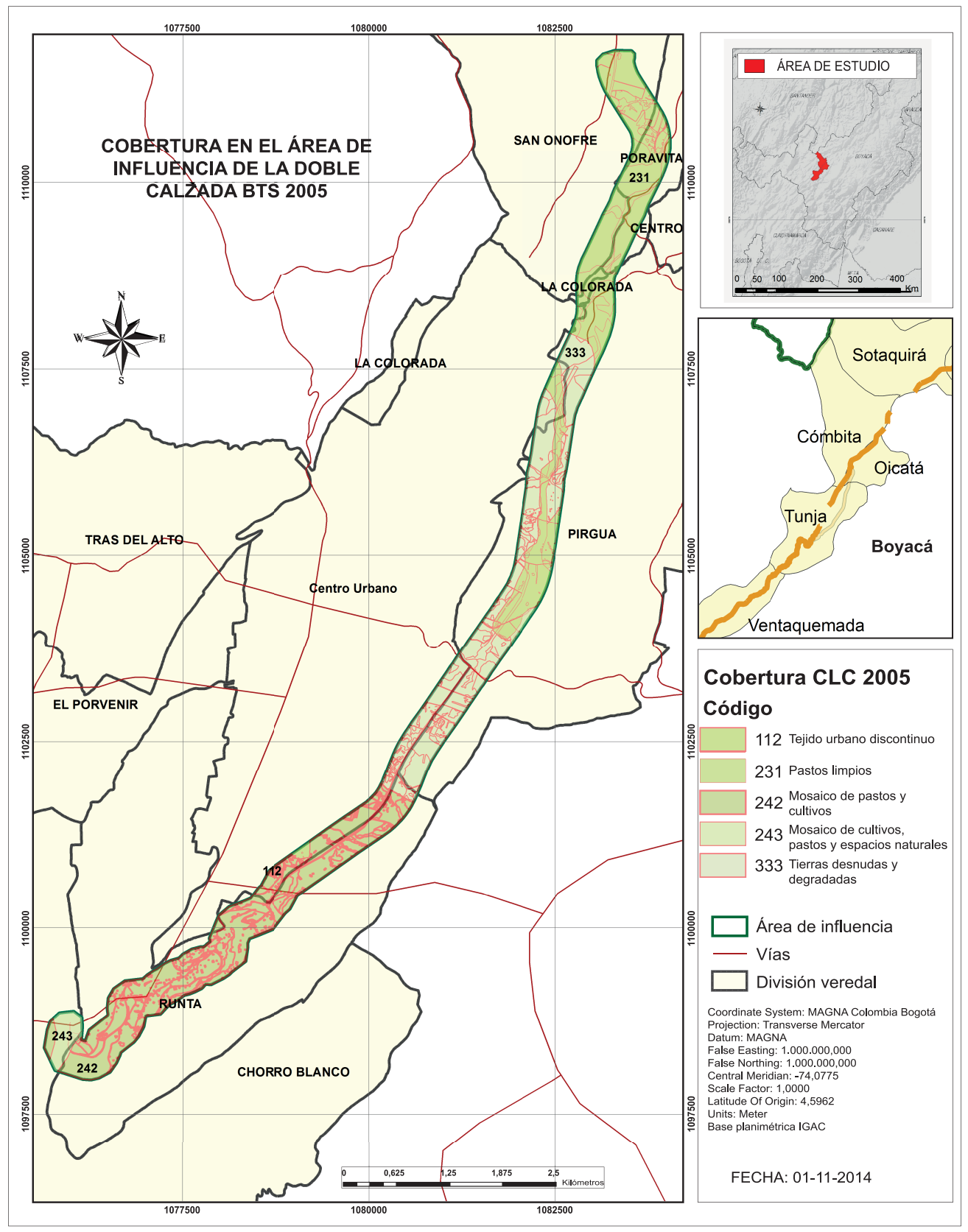

Figura 6. Cobertura del área de influencia 2005 de nivel 3 para una escala 1:100.000 Fuente: elaboración propia a partir de estudio multitemporal 2005-2012. 
Dichas categorías se localizan en su mayor parte en los extremos norte y sur del área de influencia de la vía, marcando en términos generales la incidencia especifica en áreas caracterizadas por pastos limpios y mosaico de cultivos y espacios naturales, que según el espacio físico-rural intervenido presentan mayor afectación que otras zonas del área de influencia para el año 2005.

Tabla 3. Cobertura del área de influencia 2012, del nivel 4 escala 1:10.000

\begin{tabular}{|c|c|}
\hline Categorías CLC 2012 & $\%$ \\
\hline Territorios artificializados & $5.54 \%$ \\
\hline Zonas industriales y redes de comunicación & $10.65 \%$ \\
\hline Zonas de extracción mineras y escombreras & $1.68 \%$ \\
\hline Zonas verdes artificializadas, no agrícolas & $0.37 \%$ \\
\hline Territorios agrícolas & $69.85 \%$ \\
\hline Tierras desnudas y degradadas & $11.55 \%$ \\
\hline Áreas húmedas & $0.27 \%$ \\
\hline Superficies de agua & $0.09 \%$ \\
\hline Total & $100 \%$ \\
\hline
\end{tabular}

Fuente: elaboración propia a partir de estudio multitemporal 2005-2012.

En la Tabla 3 y de acuerdo con la leyenda CLC (Ministerio de Ambiente y Desarrollo Sostenible, 2010), los territorios artificializados para el año 2012, están conformados por tejido urbano continuo y discontinuo, donde este último afectó para el año 2000-2005, el 0,1 \% de la zona urbana en expansión del municipio de Tunja. Sin embargo, para el año 2012, se aprecia un 5,54 \%, el cual permite afirmar que el área inmediata de influencia de la infraestructura vial ha alojado cambios importantes sobre su estructura espacial, debido a la localización de tejido urbano discontinuo y edificaciones rurales considerables en el corto tiempo de construida la infraestructura vial.

Las zonas industriales y redes de comunicación presentan el 10,65\% del área influenciada, caracterizada por zonas de restaurantes o zonas dedicadas al comercio, supeditadas a su vez por las relaciones orbitales de los municipios aledaños y la operación vial ofrecida por la misma infraestructura. Además se aprecia un número considerable de vías de acceso rural, que fueron el producto de la fragmentación predial y parcelamiento del área de influencia, y de la apertura de accesos viales 


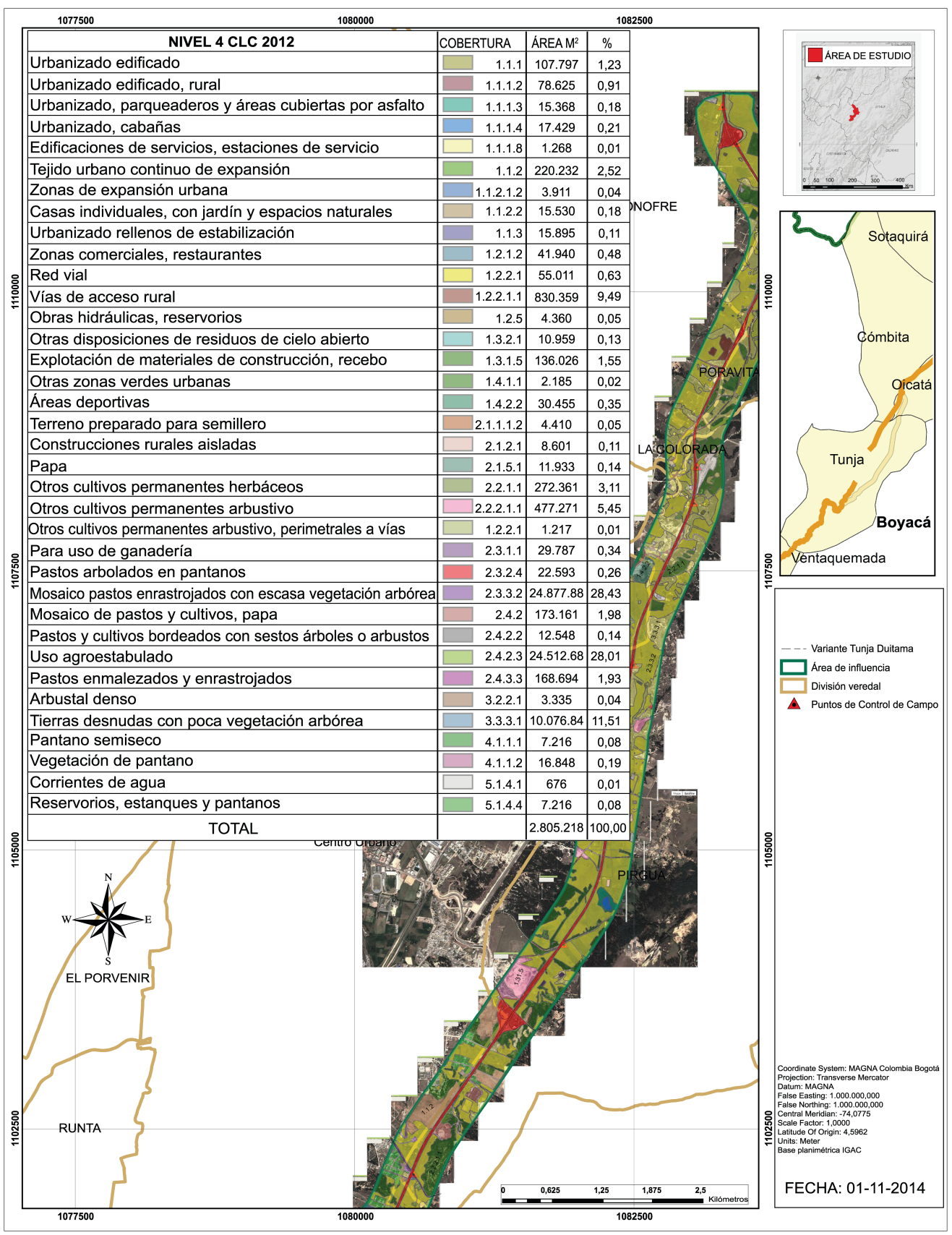

Figura 7. Cobertura del área de influencia 2012, a partir de leyenda CLC (Ministerio de Ambiente y Desarrollo Sostenible, 2010)

Fuente: elaboración propia a partir de retrointerpretación 2012. 
hacia aquellos tejidos urbanos discontinuos, como puede observarse en la Figura 7, la cual presenta el consolidado de categorías identificadas en el área de influencia para el año 2012.

Según este consolidado, dentro de las actividades de extracción, se identificaron dos tipos, la primera tiene que ver con la actividad mine$\mathrm{ra}$, y la segunda con zonas para la disposición de residuos. Estas actividades en el área de influencia corresponden al 1,68 \%, discriminado en su mayoría en zonas de extracción y explotación de materiales, que pueden corresponder a pequeños reductos localizados, que fueron parte de las zonas degradadas y desnudas encontradas en el año 2005, y que obedecen a procesos espaciales asociados por la construcción de la vía.

Ahora bien, los territorios agrícolas representan el 69,85\%, caracterizado como el área de mayor extensión y clasificación que puedo obtenerse a partir de la fotointerpretación realizada. Si se compara este porcentaje con la lectura del año 2005, refiere un $78 \%$, considerando una disminución aproximada del $10 \%$. En cuanto al componente de tierras desnudas y degradadas se obtuvo un 11,55 $\%$, cifra considerable si se tiene en cuenta que esta misma categoría para el año 2005 presentaba el $28.18 \%$, lo cual indica una disminución aproximada del $16 \%$ en estas áreas.
Una vez analizada el área de influencia de la infraestructura vial en los municipios de Tunja, Cómbita y Oicatá, se puede decir que esta área presenta una dinámica relativa en lo que respecta a desarrollo urbano y actividades comerciales, que han modificado el área de influencia al emplazarse discontinuamente en este espacio, estimulando la fragmentación de espacios naturales y la pérdida de hectáreas en cultivos que se vieron irreversiblemente afectados debido al aumento del área de expansión urbana de la ciudad de Tunja.

Así mismo, con la operación de la infraestructura vial, se observa la presencia considerable de territorios artificializados, teniendo en cuenta que esta categoría, en sus niveles 1-2-3 y 4, según la leyenda CLC (Ministerio de Ambiente y Desarrollo Sostenible, 2010), engloba el 18,24 \% del área de influencia de la vía en los municipios de Tunja, Cómbita y Oicatá, asumiendo importantes cambios en lo que respecta al año 2005. Igualmente, en lo correspondiente a las categorías de territorios agrícolas versus pastos limpios y derivados, esta se caracteriza por ser la zona de mayor influencia con una extensión del $70 \%$ aproximadamente, en la que se aprecia una disminución de tierras desnudas y degradadas en un $50 \%$.

En términos generales, los procesos espaciales asociados por la construc- 
ción de la infraestructura vial se relacionan directamente con las prácticas de uso territorial y con cada una de las dinámicas que ha conllevado su configuración espacial respecto a los sistemas viales locales y regionales a los cuales se articula, lo cual produjo nuevos accesos y cambios locales correspondientes a procesos espaciotemporales localizados y materializados según las dinámicas socioeconómicas de cada uno de los municipios.

\section{Conclusiones}

La doble calzada BTS es una obra que ha generado dinamismos localizados en el uso del suelo, los cuales han orientado nuevos procesos espaciales y han puesto de manifiesto la incidencia de la vía en la disposición de actividades y dinámicas relativas en el valor del suelo, que tienden a jalonar el desarrollo parcial y transitorio del área de influencia. Concebir como procesos espaciales asociados los posibles efectos que desarrolla este tipo de infraestructuras, permite contextualizar con mayor precisión la incidencia en términos físicos y económicos que puede albergar este tipo de obras, particularmente, al considerarlos como elementos permisivos que generan un interacción espaciotemporal en el territorio donde son incorporadas, y que en virtud de su capacidad estructurante, generan transformacio- nes en el espacio materializadas en las diferentes escalas.

Así mismo pudieron evidenciarse, frente al análisis de coberturas, cambios importantes en las categorías correspondientes a territorios agrícolas versus pastos limpios y derivados, que componen un $70 \%$ de presencia en el área de influencia, sumado a la disminución de tierras desnudas y degradas en un $50 \%$, y el aumento de territorios artificializados en un 24,8 $\%$, que indican como la construcción de este corredor vial incidió en el desarrollo espacial localizado, mediante la generación de procesos espaciales que consolidaron áreas potencialmente influyentes en las actividades socioeconómicas de los municipios influenciados.

La apuesta metodológica permitió considerar cambios en las coberturas que obedecen a la asociación de procesos no lineales en espacio y tiempo a la construcción de la infraestructura vial. El desarrollo espacial del área de influencia se ha instaurado con intensidades y frecuencias distintas, que se consolidan espacial y temporalmente de conformidad con las dinámicas económicas de los territorios intervenidos, al generar una reproducción social y material relacionada con el uso, explotación y operatividad de la infraestructura vial y de su relación y articulación con el sistema vial local de los municipios incididos. 


\section{Referencias}

Alcaldía Mayor de Tunja. (2001). Plan de ordenamiento territorial. Recuperado de: http:// cdim.esap.edu.co/BancoConocimiento/T/tunja_-_boyaca_-_pot_-_2000_-_2009/ tunja_-_boyaca_-_pot_-_2000_-_2009.asp

Bellet, C. (2013). Transporte y desarrollo territorial. El estudio de los efectos asociados a la implantación de alta velocidad ferroviaria a través del caso español. Revista Transporte y Territorio (8), 117-137. Recuperado de: http://dialnet.unirioja.es/servlet/ articulo? codigo $=4340460$

Blanco, J. (2006). De la noción de impactos a procesos asociados. Revista transporte y territorio, (3), 172-189. Recuperado de: http://www.mundourbano.unq.edu.ar/index. php/ano-2006/26-numero-28/188-2-de-la-nocion-de-impacto-a-la-de-procesos-asociados

Cerquera E. F. (2013). Análisis espacial de los accidentes de tráfico en Bogotá D.C. Fundamentos de investigación. Revista Perspectiva Geográfica, 18(1), 9-37.

Consejo Nacional de Política Económica y Social -CONPES- (2004). Modificación al Conpes 3272. Criterios para la priorización de proyectos del programa de infraestructura vial y desarrollo regional. Recuperado de: https://pwh.dnp.gov.co/LinkClick. aspx?fileticket $=$ UwafDohx-e4\%3d\&tabid $=354$

Corredor, L., Cárdenas., E. \& Ordoñez, J. (2011). Aplicación de la metodología Corine Land Cover en la determinación de los cambios de cobertura en el parque natural los flamencos. Revista Ciencia e Ingeniería Neogranadina, 21, 153-167.

Cruz, C. A. (2011). Infraestructura y conectividad en el territorio de Honduras, el proyecto Mesoamérica y su impacto local y regional Tesis de maestría. Pontificia Universidad Católica de Chile, Facultad de Arquitectura, Diseño y Estudios Urbanos, Instituto de Estudios Urbanos y Territoriales. Santiago de Chile. Recuperado de: www.estudiosurbanos.uc.cl/

Departamento Administrativo Nacional de Estadística -DANE- (2013). Metodología índice de valorización predial. Recuperado de: https://www.dane.gov.co/files/investigaciones/ fichas/Met_IVP_08_13.pdf

Estudio Socioeconómico (2011) Proyecto de concesión vial Briceño-Tunja-Sogamoso. Informe inventario socioeconómico asentado alrededor del derecho de la vía (1). Colombia.

Fariña, J., Lamiquiz, F. \& Pozueta, J. (2000). Efectos territoriales de las infraestructuras de transporte de acceso controlado. En Cuadernos de Investigación Urbanística. (pp. 10-45). Madrid, España. Recuperado de: http://polired.upm.es/index.php/ciur/article/ download/243/239 
Garmendia, M. (2008). Cambios en la estructura urbana y territorial facilitados por la alta velocidad ferroviaria. La línea Madrid-Sevilla a su paso por la provincia de ciudad real. Tesis de doctorado. Universidad de Castilla-La Mancha, España. Recuperado de https:// ruidera.uclm.es/xmlui/bitstream/handle/10578/.../TESIS.pdf?

Garmendia, M., Ureña, J. \& Coronado, J. (2011). Cambios en la estructura territorial debidos a nuevas conexiones de alta velocidad en territorios aislados: la provincia de Ciudad Real en España. Revista EURE, 37(110), 89-115.

Google Earth (s.f.). Imágenes. Recuperado de http://maps.google.com/gallery/details?id=z4fZuCLmiKg.kz3qtKM-Oia4

Governa, F. (2007). Las infraestructuras de transporte concebidas como obras territoriales. Exigencias y estrategias de territorialización. Revista Papers Instituto de estudios regionales metropolitanos de Barcelona, (44), 20-31.

Izquierdo, R. (1994). Transportes: un enfoque integral. En El transporte y su relevancia en el contexto económico y social. (pp. 53-595). España: Colegio de Ingenieros de Caminos, Canales y Puertos.

Millares-Guach, C. (2002) Trasporte y territorio urbano, del paradigma de la causalidad al de la dialéctica. Análisis Geográfico, (41), 107-120.

Ministerio de Ambiente y Desarrollo Sostenible. (2010). Leyenda nacional de coberturas de la tierra. Metodología CORINE Land Cover adaptada para Colombia escala 1:100.000. 2010. Sistema de Información Ambiental de Colombia. Colombia. Recuperado de: https://www.siac.gov.co/contenido/contenido.aspx?catID =471\&conID =647

Offner, J. M. (1993). Los «efectos estructurales» de transporte: mito político, fraude científico. Noisy-le-Grand, Francia: Escuela Nacional de Puentes y Caminos, Técnicas de Laboratorio, Territorios, Empresas, Unidad de Investigación asociada al CNRS 1245

Perdomo, J. (2010). Una propuesta metodológica para estimar cambios en el valor de la propiedad. Estudio de caso Bogotá aplicando propensity score matching y precios hedónicos espaciales. Lecturas de Economía, (73), 52-65.

Rubio, J. \& Vargas, J. (2010). Impacto de las obras públicas de infraestructura vial en los precios del suelo: una aplicación en los sectores anexos a la avenida Ciudad de Cali. Bogotá: Universidad Nacional de Colombia.

Urbano, M. (1993). Infraestructura del transporte terrestre y desarrollo regional. Tesis de doctorado. Universidad Autónoma de Madrid, Facultad Ciencias Económicas y Empresariales, Departamento de Estructura Económica y Economía del Desarrollo. Recuperado de https://repositorio.uam.es/bitstream/.../28325_martin_urbano_pablo.pdf 
Recepción: 2 de agosto de 2014

Evaluación: 12 de octubre de 2014

Aprobación: 18 de noviembre de 2014

240 Andrés Felipe Bautista Vargas, Flor Ángela Cerquera Escobar 